\title{
Avaliação do Projeto Academia da Saúde em uma determinada cidade do Norte de Minas Gerais: percepções dos participantes envolvidos
}

\author{
Evaluation of the Health Gym Project in a certain city in the North of Minas Gerais: \\ perceptions of the participants involved \\ Evaluación del Proyecto Academia de la Salud en una ciudad del Norte de Minas Gerais: \\ percepción de los participantes involucrados
}

Ana Paula Siqueira Silva ${ }^{1 *}$, Saulo Daniel Mendes Cunha², Luís Fernando Pereira da Silva .

\begin{abstract}
RESUMO
Objetivo: Analisaras percepções dos participantes envolvidos no Projeto Academia da Saúde em uma determinada cidade do norte de Minas Gerais acerca da avaliação deste projeto: benefícios para a saúde, avaliação física antes da participação, avaliação dos estagiários, pontos positivos e negativos. Métodos: Trata-se de um estudo descritivo com abordagem qualitativa, de corte transversal. A população foi composta por participantes do Projeto Academia da Saúde em uma determinada cidade do norte de Minas Gerais. A amostra foi composta por 2 grupos focais, totalizando 18 participantes. Como critérios de inclusão do estudo: participantes ativos e com participação mínima de 1 ano. A coleta de dados foi realizada através de um roteiro de entrevista semiestruturada. As informações coletadas através da entrevista foram submetidas à análise de categorias e à análise de discurso. Este projeto foi executado de acordo com resolução 466/2012 do conselho nacional de saúde. Resultados: Através dos discursos, foi possível identificar que as aulas de ginástica trouxeram benefícios aos participantes do Projeto Academia da Saúde. Conclusão: Em programas como este de atividade física é importante que todos os participantes do projeto tenham uma pré-avaliação física cautelosa, uma vez que abrange público diverso.
\end{abstract}

Palavras-chave: Políticas públicas, Saúde, Educação física.

\begin{abstract}
Objective: To analyse the perceptions of the participants involved in the Health Gym Project in a certain city in the north of Minas Gerais, about the evaluation of this project: Health benefits, Physical evaluation before participation, Evaluation of trainees, Positive and negative points. Methods: This is a descriptive study with a qualitative, cross-sectional approach. The population was composed of participants of the Health Gym Project in a certain city in the north of Minas Gerais. The sample consisted of 2 focal groups, totalling 18 participants. As criteria for inclusion of the study: active participants with a minimum participation of 1 year. Data collection was performed through a semi-structured interview script. The information collected through the interview was submitted to category analysis and discourse analysis. This project was implemented in accordance with National Health Council Resolution 466/2012.Results:Through the statements, it was possible to identify that the gym classes brought benefits to the participants of the Health Gym Project. Conclusion: In Programs like this one of physical activity it is important that all the participants of the project have a cautious physical preevaluation, since it covers diverse public.
\end{abstract}

Keywords: Public policies, Health, Physical education.

\footnotetext{
1 Educação Física, Graduação, Universidade Estadual de Montes Claros Montes Claros-Brasil. ${ }^{2}$ Professor de Educação Física da Universidade Estadual de Montes Claros (MG), Montes Claros-Brasil.

*E-mail: ana.paula.siqueira.silva@outlook.com
} 


\section{RESUMEN}

Objetivo: Analizarla percepción de los participantes involucradosen el Proyecto Academia de la Salud en determinada ciudad del norte de Minas Gerais, acerca de la evaluación de este proyecto: Beneficios para la salud, Evaluación Física antes de la participación. Evaluación de los pasantes, Puntos positivos y negativos.

Métodos: Se trata de un estudio descriptivo con abordaje cualitativo, de corte transversal. La población fuecompuesta por los participantes del Proyecto Academia de la Saluden determinada ciudad del norte de Minas Gerais. Lamuestra estuvo compuesta por 2 grupos focales, totalizando 18 participantes. Como criterios de inclusión del estudio: participantes activos ycon participación mínima de 1 año. Larecolección de los datos fue realizada através deunguión de entrevista semiestructurada. Las informaciones recolectadas través de la entrevista fueronanálizadasporcategoriasyelanálisis del discurso. Este proyecto fue ejecutado de acuerdo con la resolución 466/2012 del consejo nacional de salud. Resultados: A través de los discursos, fue posible identificar que las clases de gimnástica trajeron beneficios a los participantes del Proyecto Academia de la Salud. Conclusión: En Programas como este de actividad física es importante que todos los participantes do proyecto tengan una preevaluación física que sea cautelosa, ya que abarca un público diverso.

Palabras-clave: Políticas públicas, Salud, Educación física.

\section{INTRODUÇÃO}

As Políticas Públicas tem papel fundamental na gestão governamental pois, através destas os governantes elaboram programas, ações e atividades desenvolvidas pelo Estado diretamente ou indiretamente, com a participação de entes públicos ou privados visando assegurar direito de cidadania,(OLIVEIRA N ; SILVA D e TOSCANO J, et al., 2014).Muitos programas e ações tem como propósito a promoção da saúde, fazendo com que o indivíduo tenha responsabilidade com sua própria saúde (SOUZA,2006).

Os programas que contêm atividades físicas podem modificar o comportamento dos indivíduos, o que pode ser observado na recomendação do American College of Sports Medicine (ACSM), no qual estipula que todo cidadão deve praticar pelo menos 30 minutos de atividade física por dia, sendo distribuídos em 5 dias da semana, de forma contínua ou acumulada, (ACSM,1996). Estas pequenas quantidades de atividade física, feitas de forma regular já são capazes de trazer benefícios não só a saúde física, mas também a saúde mental e social, Organização Mundial de Saúde (OMS,1946). A prática regular de atividade física é considerada pelo Ministério da Saúde (MS), como prioridade entre as estratégias da Política Nacional de Promoção da Saúde (PNPS), tanto na rede básica, quanto por meio de ações intersetoriais e de aconselhamento e divulgação (MS, 2006).

Neste contexto de abordagem, a Secretaria de Saúde das cidades juntamente com governo federal, implantaram o Projeto Academia da Saúde em diversas cidades, tornando possível e acessível à população a prática regular de atividade física. Neste contexto essa determinada cidade do município do norte Minas Gerais implantou o Projeto Academia da Saúde no ano de 2015, em diversos bairros desta cidade. Atualmente o programa conta com 10 polos, fornecendo atividades diárias em diferentes bairros e zona rural do município. As atividades são desempenhadas de segunda a sexta-feira, nos turnos matutino e noturno, e acompanhadas por "instrutores capacitados".

Com a ampliação do projeto houve inclusão, sem distinção, de um número maior de pessoas praticando aulas de ginástica. Nesse sentido é importante avaliar este projeto através das percepções dos participantes. Tal processo se distingue em três aspectos: a efetividade é pautada nos impactos que esta população é atendida pelo programa; a eficácia determina a relação dos objetivos e os resultados deste programa e já a eficiência avalia a implementação deste programa através dos resultados obtidos (SULBRANDT J,1993).

Dentro deste contexto é necessário fazer uma avaliação sistemática deste projeto para tentar melhorar os padrões de atendimento e de funcionamento. O objetivo do presente estudo consiste em compreender a percepção dos participantes envolvidos em relação à implantação desse projeto através de análise das falas de tais, possibilitando-nos assim propriedade para constatar o nível de relevância do projeto para estas pessoas. 


\section{MÉTODOS}

O processo metodológico caracterizou-se como uma pesquisa descritiva e de corte transversal, com abordagem qualitativa. A coleta de dados foi realizada em uma determinada cidade do norte de Minas Gerais, Brasil, no mês de agosto de 2018, no Projeto Academiada Saúde. Foram realizados 2grupos focais, contendo 9 pessoas em cada grupo, realizados em dois momentos distintos, totalizando 18 participantes. Os participantes desta pesquisa foram escolhidos aleatoriamente. A população alvo do estudo contou com dezoito participantes do Projeto Academia da Saúde, sendo todas do sexo feminino, com idades que variavam entre 14 e mais 60 anos. Como critério de inclusão do estudo: participantes ativos e com participação mínima de 1 ano.

Os dados foram coletados a partir de roteiro de entrevista semiestruturada (GIL A, 2008), com duração entre uma hora e uma hora e meia, foi utilizado um gravador da marca Verde Inovação e Estilo, Gravador de Voz Digital.

Todo processo foi feito em uma sala da secretária de saúde, para preservar o anonimato das pessoas.

A transcrição dos discursos foi feita na íntegra e a partir daí, foi utilizado o método da categorização dos dados proposta por Minayo MC (2008), que tem como objetivo "agrupar elementos, ideias ou expressões em torno de um conceito capaz de abranger tudo isso". As categorias elencadas para este estudo relacionadas ao Projeto Academia da Saúde foram: Benefícios para a saúde, Avaliação Física antes da participação, Avaliação do estagiários e Pontos positivos e negativos.

A pesquisa foi aprovada pelo Comitê de Ética em Pesquisa com Seres Humanos da Universidade Estadual de Montes Claros, parecer de $\mathrm{n}^{\circ} 2.663 .413$ e aprovado com o seguinte número de CAAE 85361618.0.0000.5146 e o aceite de participação foi documentado mediante a assinatura do Termo de Consentimento Livre e Esclarecido. O projeto respeita os preceitos éticos da pesquisa em seres humanos.

\section{RESULTADOS E DISCUSSÃO}

As discussões a seguir foram norteadas de acordo com os temas elencados nos grupos focais realizados com os participantes, envolvidos neste estudo. Utilizamos sinal gráfico colchete ([...]) para recortes de algumas falas, para propiciar melhor entendimento ao texto, e por questão ética os nomes das pessoas envolvidas e da cidade pesquisada, serão preservados. Os participantes dos grupos focais foram identificados pela inicial $(\mathrm{P})$ e o número de identificação 1 ao 18. Após uma análise do material, optou-se por delimitar quatro categorias.

\section{1) Benefícios para a saúde de praticantes de ginástica}

As políticas públicas têm um grande potencial para influenciar a população a praticar atividade física. Já existem evidências que comprovam que estes programas de atividade física podem trazer benefícios para saúde, (Brasil, 2011). Neste Projeto Academia da Saúde, percebemos que alguns participantes relataram que as práticas de exercícios (durante as sessões de treino) apresentam benefícios para a saúde dos envolvidos. Segue abaixo os discursos do P1 e P4:

“....eu era muito sedentária[...]trouxe muitos benefícios para minha vida, minha saúde” (P1).

“...o projeto é excelente para mim, foi uma melhoria total na minha saúde" (P4).

De acordo com Brasil (2013) estas políticas públicas, devem ser desenvolvidas pelas cidades, para proporcionar a melhoria dos padrões de qualidade de vida e modo de vida saudáveis da população, reduzindo assim o gasto com assistência à saúde, secundária e terciária. Estas falas parecem demonstrar que estes participantes estão satisfeitos com os benefícios da atividade física para a saúde. 


\section{2) Avaliação Física dos participantes antes da entrada no Projeto Academia da Saúde}

De acordo com o Conselho Federal de Educação Física (CONFEF), avaliação física é um procedimento fundamental para o profissional de Educação Física, e tem como o principal objetivo reunir elementos para fundamentar a sua decisão, para escolha do método a ser usado para a prescrição de exercício físico, (CONFEF, 2012).

Percebemos de acordo com a fala de (P10), que avaliação física não parece ser realizada pelo profissional de Educação Física e sim pela Nutricionista. No entanto é da competência desta fazer avaliação da composição corporal, como os dados antropométricos, entretanto parece não haver uma avaliação física completa (anamnese, avaliação postural e flexibilidade...) que geralmente, é da alçada do Profissional de Educação Física. Diante disso infere-se que a participante P10 não diferencia tipos de avaliação, ou seja, considera como sendo avaliação física comum de ambos os profissionais, seja educador físico ou outro profissional da saúde. A participante relata que são avaliadas somente no início do projeto. É muito importante antes de toda atividade física, a avaliação física. Isso é necessário para constatar se o objetivo do projeto está sendo alcançado, (Schwaab, 2015). Vejamos o discurso a seguir:

... avaliação física é feita pela nutricionista[...]isso acontecia toda segunda, mas já tem um tempo que ela não faz avaliação" (P10).

Antes de entrar em qualquer programa de atividade física é essencial a realização de pré-avaliação, só assim é possível que o profissional de Educação Física libere para a prática de atividade física (CREF13/BA, 2018). Também foi relatado pelas participantes do projeto que ao adentrar no projeto, em nenhum momento foi exigido atestado médico das participantes. O sistema CREF-CONFEF reconhece que determinações legais exigem atestado médico como condição imprescindível para a prática de atividades físicas, e é de competência do Profissional de Educação Física a prescrição e orientação do exercício físico, realizadas com segurança (CREF13/BA, 2018).

\section{3) Formação e educação continuada dos professores de Educação Física atuantes no Projeto Academia da Saúde}

A competência profissional é um trabalho que requer conhecimento, experiência e vivência nas diferentes áreas do conhecimento. Sobre isto, Masetto (2008) expõem que é necessária uma formação específica e continuada aos diversos setores da organização pública e encaradas de forma profissional.

Diante desse cenário, a participante (P3) relatou que os professores estão em constante aprimoramento e conhecem a finalidade do projeto e o executam de maneira correta seguindo as orientações dos professores e coordenadores. Segue abaixo o discurso do (P3):

“...eles estão sempre estudando; e sabendo o que deve e o que não deve, [...] já estão bem treinados e capacitados o suficiente para desenvolver as atividades diárias da sua área profissional" (P3).

Desta maneira, a avaliação relatada pela participante expõe que os profissionais envolvidos neste projeto são pessoas em constante aprimoramento. Corroborando com estas ideias, Nogaro (2008) afirma que para alcançar a finalidade educativa exige-se do profissional "onde se pretende chegar" indissociáveis também "para aonde deverão seguir" dos profissionais em formação.

Percebe-se que, o profissional capacitado frente aos desafios desta proposta pública deve ser habilitado na área de atuação (Bacharel em Educação Física) e pessoal qualificado para orientações ações sobre promoção, prevenção e tratamento a saúde.

\section{4) Pontos positivos e negativos do projeto.}

Este programa apresenta aspectos relevantes à população envolvida, tanto pontos positivos quanto negativos, portanto, pensando em um cenário público, poderia este contar com todos profissionais da atenção 
básica, além de buscar parcerias, demais equipes, sendo assim mais eficaz na contribuição para melhoria de saúde dos envolvidos tendo seus resultados otimizados. Contudo, é perceptível a satisfação e evolução na melhoria da saúde do público em questão, uma vez que as atividades são adaptadas culturalmente ao território (Brasil, 2013).

De acordo com (P6), percebemos que o projeto, na visão das participantes, parece não apresentar nenhum ponto negativo, apresentando somente pontos positivos. Muitos discursos convergem para uma melhoria significativa da saúde das praticantes. Segue abaixo o discurso do (P6):

“.. nossa pra mim foi melhora total, não tem ponto negativo [...]está completo.” (P6)

Apesar das participantes não relatarem pontos negativos, em algumas falas, percebemos, através dos discursos anteriores das participantes que os profissionais parecem não fazer avaliação periódica, não há uma orientação ou atestado médico.

Outro ponto relevante também, é o fato do profissional de Educação Física que orienta as participantes, não fazer a avaliação física, ademais, como este projeto propicia satisfação a seus participantes, tais aspectos acabam sendo sobrepostos pelos benefícios que proporciona. De acordo com a fala da participante (P6) parece demonstrar que está satisfeita com este projeto.

\section{CONSIDERAÇÕES FINAIS}

Em Programas como este de atividade física é importante que todos os participantes do projeto tenham uma pré-avaliação física seja cautelosa, uma vez que abrange público diverso. Os profissionais envolvidos na avaliação física, prescrição e acompanhamento devem-se atentar para os elementos importantes na manutenção da segurança e integridade das participantes. Identificamos através deste estudo que à avaliação física deveria ser feita pelo profissional habilitado. Apesar disso, corroboramos a pertinência do projeto e os benefícios que este proporciona a seus participantes, cumprindo seu maior objetivo que é a melhoria da qualidade de vida de seus participantes.

\section{REFERÊNCIAS}

1. ACSM. American College of Sports Medicine. Guidelines for graded exercise testing and exercise prescription. Philadelphia: Lea \&Febiger, 1986.

2. Brasil. Avaliação de efetividade programas de atividade física no Brasil. Brasília: Ministério da Saúde, 2011; 184p.

3. Brasil. Redefine o Programa Academia da Saúde no âmbito do Sistema Único de Saúde (SUS). Diário Oficial da União 2013; 8 nov.

4. CONFEF. Nota técnica N002/2012. Disponível em:https://www.confef.org.br/confef/conteudo/837.Acesso em : 3 de fev.2019

5. CREF13/BA. Nota técnica No 002/2018. Disponível em: http://www.cref13.org.br/bahia/wpcontent/uploads/2018/06/NOTA-T\%C3\%89CNICA-N\%C2\%BA-002.2018-AVALIA\%C3\%87\%C3\%83O-PREPARTICIPA\%C3\%87\%C3\%8301. Acesso em: 3 de fev.2019.

6. FILHO, José Fernandes. A prática da Avaliação Física.Shape,2003.

7. GIL AC. Métodos e técnicas de pesquisa social. Atlas, 2008; p. 26-28.

8. Masetto MT. Docência na Universidade:Professor universitário: Um profissional da educação na atividade docente. Campinas: Papirus, 2008b.

9. MINAYO MC. O Desafio do Conhecimento: pesquisa qualitativa em saúde. São Paulo: Hucitec, 2008.

10. MS. Secretaria de Vigilância em Saúde. Secretaria de Atenção à Saúde. Política Nacional de Promoção da Saúde. Brasília: Ministério da Saúde; 2006.

11. Nogaro A. Aprender-desaprender-reaprender a dinâmica da aula universitária. Unochapecó, Revista Pedagógica, 2008; v. 10, n. 20, p. 1-8,jan./jun.

12. OLIVEIRAN ; SILVA D e TOSCANO J, et al., Programas de avaliação em academias de ginástica: o que se faz? Revista Brasileira de Atividade, 2014.

13. OMS. Organização Mundial de Saúde.Carta de Constituição da Organização Mundial de Saúde. Nova York. 1946.

14. SCHWAAB F. A influência da avaliação antropométrica na mudança do estilo de vida dos sujeitos praticantes de musculação ljuí -RS. 2015.

15. SULBRANDT J. La evaluación de los programas sociales: una perspectiva crítica de los modelos usuales. In: KLIKSBERG, B. Pobreza: un tema impostergable. México: Fondo de Cultura Económica, 1993.

16. SOUZA C. Políticas públicas: uma revisão da literatura. Sociologias, Porto Alegre jul./dez. 2006; n.16. 